\title{
Genetic Profile Assessment of Giant Clam Genus Tridacna as a Basis for Resource Management at Wakatobi National Park Waters
}

\author{
Muhammad Nur Findra ${ }^{1 *}$, Isdradjad Setyobudiandi ${ }^{2}$, Nurlisa A. Butet ${ }^{2}$ and Dedy Duryadi Solihin ${ }^{3}$ \\ 1 Graduate Program of Aquatic Resources Management, Bogor Agricultural University \\ J. Agatis, Kampus IPB Dramaga Bogor 16680, Indonesia \\ ${ }^{2}$ Department of Aquatic Resources Management, Faculty of Fisheries and Marine Science, \\ Bogor Agricultural University \\ J. Agatis, Kampus IPB Dramaga Bogor 16680, Indonesia \\ ${ }^{3}$ Department of Biology, Faculty of Mathematics and Science, Bogor Agricultural University, \\ J. Agatis, Kampus IPB Dramaga Bogor 16680, Indonesia \\ Email: muhammad.findra@gmail.com
}

\begin{abstract}
Giant clam population has been decreased in a few years. Resource management requires information from various aspects, such as ecological, population, and other aspects. This study was aimed at assessing the genetic profile of Tridacna giant clam in Wakatobi National Park waters using Cytochrome oxidase subunit I (COI) genetic marker. Sample collection was conducted around the three main islands, i.e., Wangi-wangi, Kaledupa, and Tomia. Genetic analysis using $\mathrm{COl}$ gene may contribute in identifying giant clams up to the species level and showed the relationship among species. The research found 41 specific nucleotide sites for the clams. T. crocea, $T$. squamosa and T. maxima had 2, 15 and 24 sites, respectively. COl gene as a biological marker was able to separate groups of giant clam by species. Nucleotide variation of T. crocea from Wakatobi was the highest among other locations, so it could be used as a genetic source for translocation and domestication.
\end{abstract}

Keywords: cytochrome oxidase subunit I, specific nucleotide, Tridacna, Wakatobi National Park

\section{Introduction}

Giant clam is one of the bivalve molluscs (Cardiidae, Tridacninae) inhabiting coral reefs and its surroundings. It is attached to coral reefs, as well as burried on sandy substrate on the reefs and seagrass beds (Knop, 1996). Giant clam population has been decreased. Fishing is the main factor of the decline of the wild giant clam population (ShauHwai and Yasin, 2003; Romimohtarto and Juwana, 2005; Larrue, 2006). Their commercial appeal encourage the intensively use of clam (shell and meat) by fishermen; therefore, exceeding the carrying capacity of the population in the wild (Panggabean, 1991). Because of the decreased population and the critical need to maintain their sustainability, they had been included in the list of protected biota based on Government Regulation (PP) No. 7 of 1999.

Seven out of eleven species of giant clams of the world inhabit Indonesian waters. Those seven species belong to of two generas, i.e., Tridacna and Hippopus. There are five species of Tridacna, i.e., Tridacna gigas, T. derasa, T. squamosa, T. maxima, T. crocea; while Hippopus consists two species, i.e., Hippopus hippopus and $H$. porcellanus (Mudjiono,
1988; bin Othman et al., 2010; Hernawan, 2012). Other species that are not found in Indonesia are $T$. tevoroa (Lucas et al., 1990), T. rosewateri (Sirenko and Scarlato, 1991), T. costata (Richter et al., 2008) and T. ningaloo (Penny and Willan, 2014).

Genus Tridacna is generally facilitated with mantle, with attractive and flashy colors over the edge of shell (Calumpong, 1992). Their outer shell shape can be divided into two groups, with shell scales, i.e., T. squamosa, T. maxima and T. crocea and groups with a shell without scales, i.e., $T$. derasa and $T$. gigas (Knop, 1996). They also have sizes from small to large. The largest size $(>100 \mathrm{~cm})$ can be found in this genus, namely $T$. gigas.

One of their distribution areas in Indonesia is Wakatobi National Park waters. Wakatobi National Park is administratively located in Wakatobi of Southeast Sulawesi province. It is defined by the government as a national park by decree of the Minister of Forestry No. 7651/ Kpts-II/2002, and is managed by a zoning system. There are at least five species found there, namely $T$. crocea, $T$. squamosa, T. maxima, T. gigas and H. hippopus (Findra, 2010).

Resource management requires information from various aspects, both biological, population and 
others, so it would be more focused and successful. The genetic information is one of the aspect that is needed in the management and conservation. In Indonesia, it is still less noticeable, whereas the role of adaptation and animal development strategy is largely determined by genetic capabilities. Genetic diversity information can be obtained by analyzing the protein-coding genes of mitochondrial DNA. Part of them often used in species study and animal population is Cytochrome oxidase subunit I (COI) (Solihin, 1994).

$\mathrm{COI}$ is a gene that evolved very slowly so it can be used as DNA barcoding (Hebert et al., 2003). It is an efficient method for the spesies identification and has a role in biodiversity taxonomic and population genetics study (Hajibabaei et al., 2007). Studies using $\mathrm{COI}$ genes as genetic markers of the giant clams have been conducted few years ago by Nuryanto et al. (2007) in several places in Indonesia, Tisera et al. (2012) in Savu Sea East Nusa Tenggara, Lizano and Santos (2014) in the Philippines. However, giant clam population of Wakatobi waters has not been genetically identified. Therefore, this study was aimed at assessing the genetic profile of giant clams using $\mathrm{COI}$ genetic markers especially genus Tridacna in the Wakatobi National Park as a data for resource management.

\section{Materials and Methods}

Giant clams were collected from Wakatobi National Park waters around the three main islands, i.e., Wangi-wangi, Kaledupa and Tomia (Figure 1.). Analysis of samples carried out in Laboratory of Animal Biomolecular, Research Center for Biological Resources and Biotechnology (PPSHB) and the Integrated Laboratory of the Department of Biology, Bogor Agricultural University.

Sample of each species identified based on the description by Knop (1996) was collected from each sampling location. Samples were taken from mantle tissue using scissors. Samples were inserted into the tube and then preserved using 96\% alcohol. (Table 1).

\section{Total DNA Isolation and Extraction}

Prior to isolation and extraction total DNA, samples were washed using Low TE so that the sample free of alcohol as a preservative. Further samples were isolated using a commercial kit from GeneAid. Its procedures performed following the manual from the factory with some procedures that had been accordingly modified.

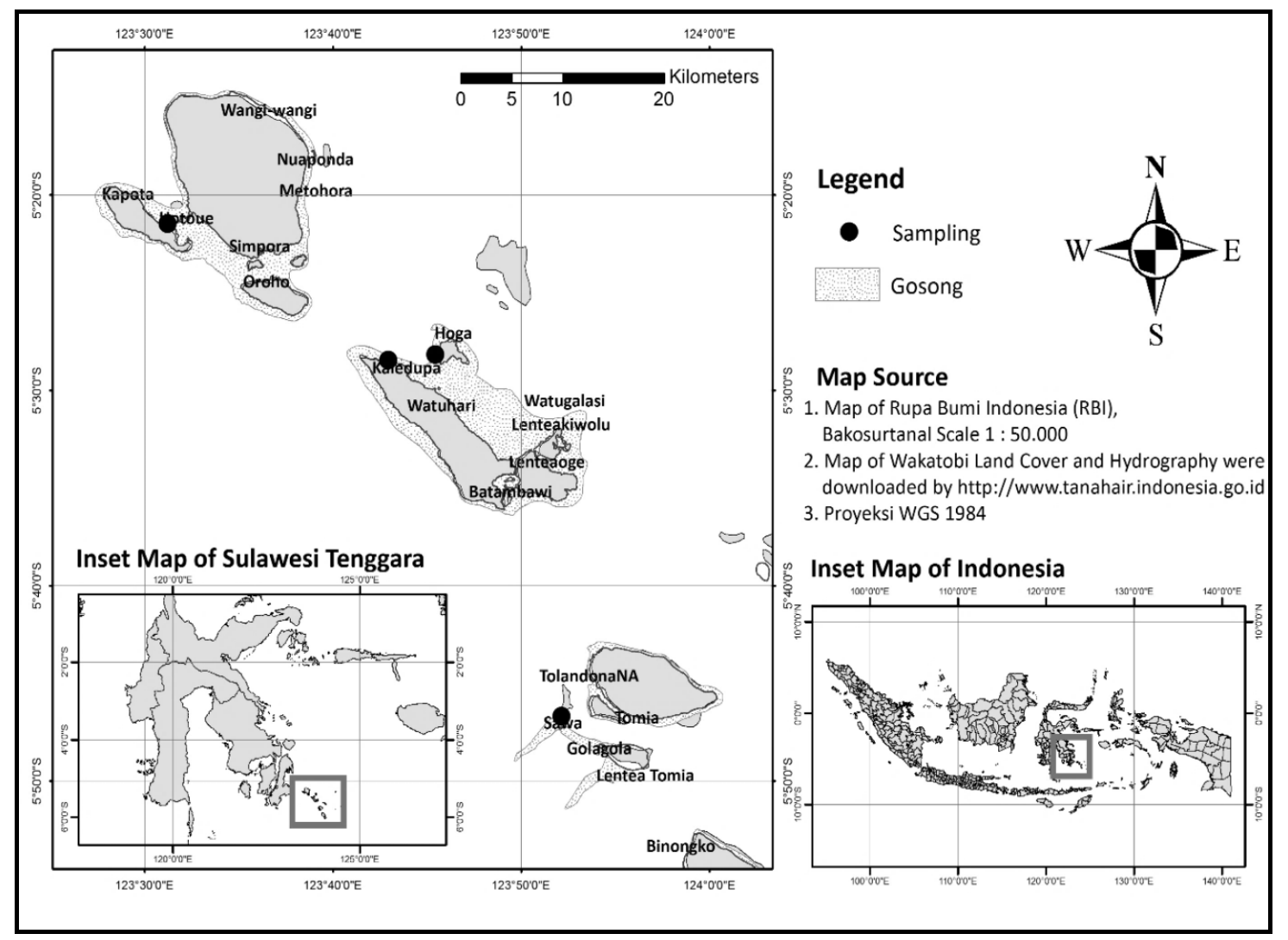

Figure 1. Map of sampling locations at Wakatobi National Park, Sulawesi. 


\section{Target Gene Amplification, Sequencing and Data Analysis}

Target gene segment was amplified using PCR (Polymerase Chain Reaction). Amplification used specific primers for tridacnid that had been designed by Nuryanto et al. (2007), LCO: 5'-GGG GAA TTC TAA TGA CAG AA-3 'and RCO: 5'-TAG TTA CAG CTA CCC AAG AA-3'. The reaction total volume was $25 \mathrm{ml}$ consisting of $9.8 \mathrm{ml}$ of $\mathrm{dd}_{2} \mathrm{O}, 4 \mathrm{ml}$ Q5 buffer, Q5 enhancher $5 \mathrm{ml}, 1 \mathrm{ml}$ of dNTP, $1 \mu \mathrm{l}$ forwards primer, reverse primer $1 \mathrm{ml}, 3 \mathrm{ml}$ of DNA template and $0.2 \mathrm{ml}$ of Q5 Taq Hot Start.

Amplification was conducted under predenaturation $95^{\circ} \mathrm{C}$ for 5 minutes, followed by 35 cycles consisting of denaturation $94^{\circ} \mathrm{C}$ for 45 seconds; annealing $49^{\circ} \mathrm{C}$ for $T$. crocea and $T$. maxima, and $52^{\circ} \mathrm{C}$ for $T$. squamosa for 45 seconds; and extension $72^{\circ} \mathrm{C}$ for 1 minute and final extension of $72^{\circ} \mathrm{C}$ for 7 minutes. The amplicons were tested electrophoresis using $1.2 \%$ agarose gel in $1 \mathrm{X}$ TBE (Tris-borate-EDTA) buffer. PCR products were either single band seen during electrophoresis (size 522 $\mathrm{bp}$ ) proceed to the stage of sequencing to look at the sequence of nucleotide. They were sent to 1st BASE Sequencing, Malaysia.

Sequences were corrected and aligned using software MEGA 5.0 (Tamura et al., 2011). Sequences from each sample was BLAST in GenBank to determine the proximity to other sequences stored in GenBank. The closeness became secondary sequence data to be analyzed to produce phylogenetic tree. Its reconstruction used Neighbour Joining method with p-distance model, 1000 bootstrap replicates. These analysis used several sequences from GenBank as their ingroup and outgroup. They were $T$. crocea from Spermonde Islands (Accession EU003606), T. crocea from Seribu Island (Accession EU003608), T. maxima from Padang (Accession EU003610), T. maxima from Biak (Accession EU003613), T. squamosa from Philippines (Accession KJ202117) and H. hippopus from Philippines (Accession KJ202106).

\section{Results and Discussion}

There were seven successfully amplified sequences (from 24 samples) using specific primers, while the others were not successfully amplified. It could be due to unsuccess DNA extraction process. The failure was thought to be caused by the presence of impurities in the form of residual preservatives and mucus, as well as their algal symbionts zooxanthellae contained in the mantle tissue of clams. The similar case was reported by Haerul (2014), that symbionts algae became impurities in DNA extraction process.

The sequences were successfully traced the nucleotides derived from three species, i.e., $T$. crocea, T. squamosa and T. maxima. The forward and reverse sequences of each individual were combined and aligned, so we obtained the nucleotide size of \pm 522 bp (Figure 2 ).

Several samples that morphologically identified as $T$. squamosa, following validation using BLAST that those performed different species (Table 2). TsH 1 and TsS 1 were originally identified as $T$. squamosa after BLAST indicated that they were $T$. crocea and T. maxima, respectively. In aquatic organism, we often found cryptic species phenomenon, which was morphologically similar, however; it was different in genetics. It could lead to false identification (Bickford et al., 2006). This study showed that giant clams were cryptic species, their morphologies were alike among distinct species, so often misidentified by their morphology. These three are included in a group which have scales and their shells are generally embedded in part or whole in

Table 1. Species and number of samples collection

\begin{tabular}{|c|c|c|c|c|}
\hline No. & Species & Location & Code & Number \\
\hline \multirow[t]{3}{*}{1.} & T. crocea & Langgira, Kaledupa & TCL & 2 \\
\hline & & Hoga Island, Kaledupa & $\mathrm{TcH}$ & 3 \\
\hline & & Sawa Island, Tomia & TcS & 1 \\
\hline \multirow[t]{4}{*}{2.} & T. squamosa & Kapota Island, Wangi-wangi & TsW & 1 \\
\hline & & Langgira, Kaledupa & TsL & 5 \\
\hline & & Hoga Island, Kaledupa & $\mathrm{TsH}$ & 5 \\
\hline & & Sawa Island, Tomia & TsS & 1 \\
\hline \multirow[t]{4}{*}{3.} & T. maxima & Langgira, Kaledupa & $\mathrm{TmL}$ & 1 \\
\hline & & Hoga Island, Kaledupa & $\mathrm{TmH}$ & 3 \\
\hline & & Sawa Island, Tomia & TmS & 1 \\
\hline & \multicolumn{2}{|c|}{ Total } & & 24 \\
\hline
\end{tabular}




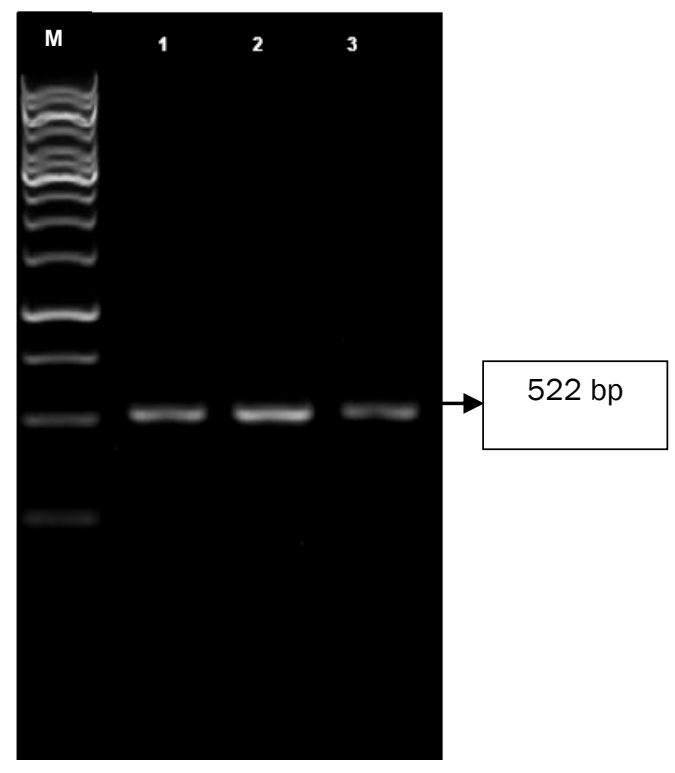

Figure 2. PCR products successfully amplified using COI gene showing intact bands $(M=1 \mathrm{~kb}$ DNA Ladder; $1=T$. crocea; $2=T$. squamosa; $3=T$. maxima)

the reef, so only mantle was clearly shown up. In addition, samples taken in this study were still juvenile, so it was difficult to identify up to species level. This study also proved that molecular analysis using COI genetic markers may contribute in identifying giant clams up to the species level.

Alignment of seven $\mathrm{COI}$ gene sequences consisting of four, one and two sequences $T$. crocea, $T$. squamosa and T. maxima, respectively, showed that few variable sites (17.82\%), while conserve sites as many as $82.18 \%$. According to Herbert et al. (2003), COI gene has conservative nucleotide base composition with a bit insertions, deletions and variations so that is used as DNA barcoding.

This research found 41 specific nucleotide sites, T. crocea, T. squamosa and T. maxima had 2, 15, and 24 sites, respectively (Table 3 ). Those nucleotide sites were the specific genetic marker which can differentiate those species.
The genetic distance intra-species was less than $2 \%$ and inter-species was more than $5 \%$ (Table 4). T. crocea, T. squamosa and T. maxima had genetic distance of $1.43 \%, 0.93 \%$, and $1.40 \%$, respectively. Genetic distance of less than or equal to $3 \%$ could be said similar species, while the genetic distance of more than $3 \%$ showed different species. According to Ratnasingham and Hebert (2013), COI gene variations of more than $4 \%$ are close relatives and isolated reproduction, if the difference of less than $2 \%$ are the same species (intra-species).

The phylogenetic tree showed that generally formed two clades (Figure 3): the first consisted of $T$. crocea, $T$. squamosa and $T$. maxima, the second clade was $H$. hippopus. It indicated that all species of the genus Tridacna were monophyletic and separated from $H$. hippopus which was another genus. Phylogenetic tree reconstructed by Nuryanto et al. (2007) using the Neighbour Joining method also showed the same phenomenon, that all species of the genus Tridacna was monophyletic. T. crocea and $T$. squamosa were in the same subclade, while $T$. maxima and $T$. gigas were in other subclade. Phylogenetic tree reconstructed by Lizano and Santos (2014) showed the same trend, T. crocea and $T$. squamosa were also at the same subclade. But, there was a difference between Nuryanto et al. (2007) and Lizano and Santos (2014). Phylogenetic tree reconstructed by Nuryanto et al. (2007) showed that $T$. gigas and $T$. maxima were in one group and sister taxa with group of $T$. crocea and $T$. squamosa, whereas phylogenetic tree reconstructed by Lizano and Santos (2014) showed that $T$. gigas separately clustered from group of $T$. crocea, $T$. squamosa and T. maxima. Reconstruction of phylogenetic tree using 16S rRNA gene fragment by Schneider and O'Foighil (1999) also showed that all species of the genus Tridacna were monophyletic, as well as the genus Hippopus. Tridacna clade formed two groups, the first group consisted of $T$. tevoroa $(T$. gigas $+T$. derasa), and the second group consisted of $T$. maxima ( $T$. squamosa $+T$. crocea). Reconstruction of the phylogenetic tree using either $\mathrm{COI}$ or $16 \mathrm{~S}$ rRNA gene fragment showed consistent results for $T$. crocea, T. squamosa and T. maxima. They were

Table 2. Results of nucleotide bases BLAST in GenBank

\begin{tabular}{cccccc}
\hline No. & Sample & Query Cover & Identity & Species Validation & Accession \\
\hline 1 & TcL 1 & $100 \%$ & $99 \%$ & T. crocea & DQ269479.1 \\
2 & TcH 2 & $100 \%$ & $98 \%$ & T. crocea & DQ269479.1 \\
3 & TcS 1 & $100 \%$ & $98 \%$ & T. crocea & DQ269479.1 \\
4 & TsW 1 & $98 \%$ & $97 \%$ & T. squamosa & KP205428.1 \\
5 & TsH 1 & $100 \%$ & $98 \%$ & T. crocea & DQ269479.1 \\
6 & TsS 1 & $97 \%$ & $99 \%$ & T. maxima & DQ155301.2 \\
7 & TmH 1 & $98 \%$ & $99 \%$ & T. maxima & DQ155301.2 \\
\hline
\end{tabular}


Table 3. Specific nucleotides of giant clam species

\begin{tabular}{|c|c|c|c|c|c|c|c|c|c|c|c|c|c|c|}
\hline \multirow{2}{*}{ Species } & \multicolumn{14}{|c|}{ Nucleotide position } \\
\hline & 9 & 16 & 18 & 28 & 29 & 30 & 36 & 42 & 45 & 87 & 90 & 105 & 108 & 150 \\
\hline T. crocea Wakatobi 1 & $T$ & $T$ & $\mathrm{G}$ & $\mathrm{G}$ & C & $\mathrm{C}$ & $\bar{T}$ & A & $T$ & A & A & $T$ & $T$ & $A$ \\
\hline T. crocea Wakatobi 2 & $\mathrm{~T}$ & $\mathrm{~T}$ & G & G & C & C & $\mathrm{T}$ & A & $\mathrm{T}$ & A & A & $\mathrm{T}$ & $\mathrm{T}$ & A \\
\hline T. crocea Wakatobi 3 & $\mathrm{~T}$ & $\mathrm{~T}$ & $\mathrm{G}$ & G & C & C & $\mathrm{T}$ & A & $\mathrm{T}$ & A & A & $\mathrm{T}$ & $\mathrm{T}$ & A \\
\hline T. crocea Wakatobi 4 & $\mathrm{~T}$ & $\mathrm{~T}$ & $\mathrm{G}$ & G & C & C & $\mathrm{T}$ & A & $\mathrm{T}$ & A & A & $\mathrm{T}$ & $\mathrm{T}$ & A \\
\hline T. crocea Spermonde GB* & $\mathrm{T}$ & $\mathrm{T}$ & G & G & C & C & $\mathrm{T}$ & A & $\mathrm{T}$ & A & A & $\mathrm{T}$ & $\mathrm{T}$ & A \\
\hline T. crocea Seribu Island GB* & $\mathrm{T}$ & $\mathrm{T}$ & $G$ & G & C & C & $\mathrm{T}$ & A & $\mathrm{T}$ & A & A & $\mathrm{T}$ & $\mathrm{T}$ & A \\
\hline T. squamosa Wakatobi & C & $\mathrm{T}$ & A & G & C & C & C & G & $\mathrm{T}$ & A & A & C & C & A \\
\hline T. squamosa Filipina GB* & C & $\mathrm{T}$ & A & G & C & C & C & G & $\mathrm{T}$ & A & A & C & C & A \\
\hline T. maxima Wakatobi 1 & $\mathrm{~T}$ & C & G & A & $\mathrm{G}$ & A & $\mathrm{T}$ & A & C & G & G & $\mathrm{T}$ & $\mathrm{T}$ & $\mathrm{G}$ \\
\hline T. maxima Wakatobi 2 & $\mathrm{~T}$ & C & $G$ & A & $G$ & A & $\mathrm{T}$ & A & C & G & $G$ & $\mathrm{~T}$ & $\mathrm{~T}$ & $G$ \\
\hline T. maxima Padang GB* & $\mathrm{T}$ & C & $\mathrm{G}$ & A & G & A & $\mathrm{T}$ & A & C & G & G & $\mathrm{T}$ & $\mathrm{T}$ & G \\
\hline T. maxima Biak GB* & $T$ & C & $\mathrm{G}$ & A & G & A & $\mathrm{T}$ & A & C & G & G & $\mathrm{T}$ & $\mathrm{T}$ & G \\
\hline \multirow{2}{*}{ Species } & \multicolumn{14}{|c|}{ Nucleotide position } \\
\hline & 156 & 168 & 172 & 174 & 189 & 192 & 205 & 208 & 216 & 222 & 238 & 249 & 258 & 268 \\
\hline T. crocea Wakatobi 1 & $\mathrm{C}$ & C & C & A & $\mathrm{G}$ & A & $T$ & C & G & G & $\mathrm{G}$ & $\mathrm{T}$ & $\mathrm{C}$ & $\mathrm{T}$ \\
\hline T. crocea Wakatobi 2 & C & C & C & A & G & A & $\mathrm{T}$ & C & G & G & G & $\mathrm{T}$ & C & $\mathrm{T}$ \\
\hline T. crocea Wakatobi 3 & C & C & C & A & G & A & $\mathrm{T}$ & C & G & $\mathrm{G}$ & G & $\mathrm{T}$ & C & $\mathrm{T}$ \\
\hline T. crocea Wakatobi 4 & C & C & C & A & $G$ & A & $\mathrm{T}$ & C & $G$ & $\mathrm{G}$ & $G$ & $\mathrm{~T}$ & C & $\mathrm{T}$ \\
\hline T. crocea Spermonde GB* & C & C & C & A & G & A & $\mathrm{T}$ & C & G & G & G & $\mathrm{T}$ & C & $\mathrm{T}$ \\
\hline T. crocea Seribu Island GB* & C & C & C & A & $G$ & A & $\mathrm{T}$ & C & $G$ & G & $G$ & $\mathrm{~T}$ & C & $\mathrm{T}$ \\
\hline T. squamosa Wakatobi & $\mathrm{T}$ & C & C & $A$ & $\mathrm{G}$ & A & G & C & $\mathrm{G}$ & G & G & C & $\mathrm{T}$ & $\mathrm{T}$ \\
\hline T. squamosa Filipina GB* & $\mathrm{T}$ & C & C & A & $\mathrm{G}$ & A & G & C & $G$ & G & $G$ & C & $\mathrm{T}$ & $\mathrm{T}$ \\
\hline T. maxima Wakatobi 1 & C & $\mathrm{T}$ & $\mathrm{T}$ & G & A & $\mathrm{T}$ & $\mathrm{T}$ & $\mathrm{T}$ & A & A & A & $\mathrm{T}$ & C & C \\
\hline T. maxima Wakatobi 2 & C & $\mathrm{T}$ & $\mathrm{T}$ & G & A & $\mathrm{T}$ & $\mathrm{T}$ & $\mathrm{T}$ & A & A & A & $\mathrm{T}$ & C & C \\
\hline T. maxima Padang GB* & C & $\mathrm{T}$ & $\mathrm{T}$ & G & $A$ & $\mathrm{~T}$ & $\mathrm{~T}$ & $\mathrm{~T}$ & A & A & A & $\mathrm{T}$ & C & C \\
\hline T. maxima Biak GB* & $\mathrm{C}$ & $\mathrm{T}$ & $\mathrm{T}$ & G & $\mathrm{A}$ & $\mathrm{T}$ & $\mathrm{T}$ & $\mathrm{T}$ & A & A & A & $\mathrm{T}$ & C & C \\
\hline \multirow{2}{*}{ Species } & \multicolumn{14}{|c|}{ Nucleotide position } \\
\hline & 270 & 282 & 286 & 297 & 306 & 339 & 342 & 354 & 357 & 360 & 366 & 372 & 417 & \\
\hline T. crocea Wakatobi 1 & $\mathrm{G}$ & $T$ & $\mathrm{C}$ & 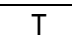 & $\mathrm{T}$ & $\mathrm{T}$ & $T$ & $T$ & $\mathrm{G}$ & $\mathrm{G}$ & $\mathrm{C}$ & $\mathrm{G}$ & $\mathrm{C}$ & \\
\hline T. crocea Wakatobi 2 & G & $\mathrm{T}$ & C & $\mathrm{T}$ & $\mathrm{T}$ & $\mathrm{T}$ & $\mathrm{T}$ & $\mathrm{T}$ & $\mathrm{G}$ & $\mathrm{G}$ & C & G & C & \\
\hline T. crocea Wakatobi 3 & G & $\mathrm{T}$ & C & $\mathrm{T}$ & $\mathrm{T}$ & $\mathrm{T}$ & $\mathrm{T}$ & $\mathrm{T}$ & $\mathrm{G}$ & A & $\mathrm{T}$ & $G$ & C & \\
\hline T. crocea Wakatobi 4 & G & $\mathrm{T}$ & C & $\mathrm{T}$ & $\mathrm{T}$ & $\mathrm{T}$ & $\mathrm{T}$ & $\mathrm{T}$ & $\mathrm{G}$ & G & C & G & C & \\
\hline T. crocea Spermonde GB* & G & $\mathrm{T}$ & C & $\mathrm{T}$ & $\mathrm{T}$ & $\mathrm{T}$ & $\mathrm{T}$ & $\mathrm{T}$ & $\mathrm{G}$ & A & $\mathrm{T}$ & G & C & \\
\hline T. crocea Seribu Island GB* & G & $\mathrm{T}$ & C & $\mathrm{T}$ & $\mathrm{T}$ & $\mathrm{T}$ & $\mathrm{T}$ & $\mathrm{T}$ & $\mathrm{G}$ & A & $\mathrm{T}$ & G & C & \\
\hline T. squamosa Wakatobi & A & C & C & $\mathrm{T}$ & C & $\mathrm{T}$ & $\mathrm{T}$ & $\mathrm{T}$ & A & A & $\mathrm{T}$ & A & C & \\
\hline T. squamosa Filipina $\mathrm{GB}^{*}$ & A & C & C & $\mathrm{T}$ & C & $\mathrm{T}$ & $\mathrm{T}$ & $\mathrm{T}$ & A & A & $\mathrm{T}$ & A & C & \\
\hline T. maxima Wakatobi 1 & G & $\mathrm{T}$ & $\mathrm{T}$ & G & $\mathrm{T}$ & C & A & C & $\mathrm{G}$ & A & $\mathrm{T}$ & G & $\mathrm{T}$ & \\
\hline T. maxima Wakatobi 2 & G & $\mathrm{T}$ & $\mathrm{T}$ & G & $\mathrm{T}$ & C & A & C & $G$ & A & $\mathrm{T}$ & G & $\mathrm{T}$ & \\
\hline T. maxima Padang $\mathrm{GB}^{*}$ & G & $\mathrm{T}$ & $\mathrm{T}$ & G & $\mathrm{T}$ & C & A & C & $\mathrm{G}$ & A & $\mathrm{T}$ & $G$ & $\mathrm{~T}$ & \\
\hline T. maxima Biak GB* & G & $\mathrm{T}$ & $\mathrm{T}$ & G & $\mathrm{T}$ & C & A & C & $\mathrm{G}$ & A & $\mathrm{T}$ & G & $\mathrm{T}$ & \\
\hline
\end{tabular}

* GB = GenBank Data

T. crocea Wakatobi 1 from Langgira, Kaledupa; T. crocea Wakatobi 2 and 4 from Hoga Island, Kaledupa; T. crocea Wakatobi 3 from Sawa Island, Tomia; T. squamosa Wakatobi from Kapota Island, Wangi-wangi; T. maxima Wakatobi 1 from Hoga Island, Kaledupa; T. maxima Wakatobi 2 from Sawa Island, Tomia.

included in subgenus Chametrachea. According to Hernawan (2012), genus Tridacna consisted of three subgenus, namely Tridacna sensu strict, Persikima and Chametrachea. Subgenus Tridacna strict sensu included only $T$. gigas, subgenus Persikima consisted of $T$. derasa and $T$. tevoroa, subgenus Chametrachea composed of $T$. squamosa, $T$. crocea, T. maxima, T. costata, and T. rosewateri.

The phylogenetic tree also showed that $T$. crocea formed two groups, the first group consisted of T. crocea Spermonde, T. crocea Seribu Island and T. crocea Wakatobi 3, while the second group consisted of $T$. crocea Wakatobi 1, T. crocea Wakatobi 2 and T. crocea Wakatobi 4. Wakatobi $T$. crocea was highly varied, because it possesses not only similar nucleotide sequences of Spermonde and Seribu Island $T$. crocea but also specific nucleotide sequences Wakatobi $T$. crocea itself (Table 5). It was due to varying environmental characteristics in these waters. Difference in habitat typology and geographically isolated would lead to 


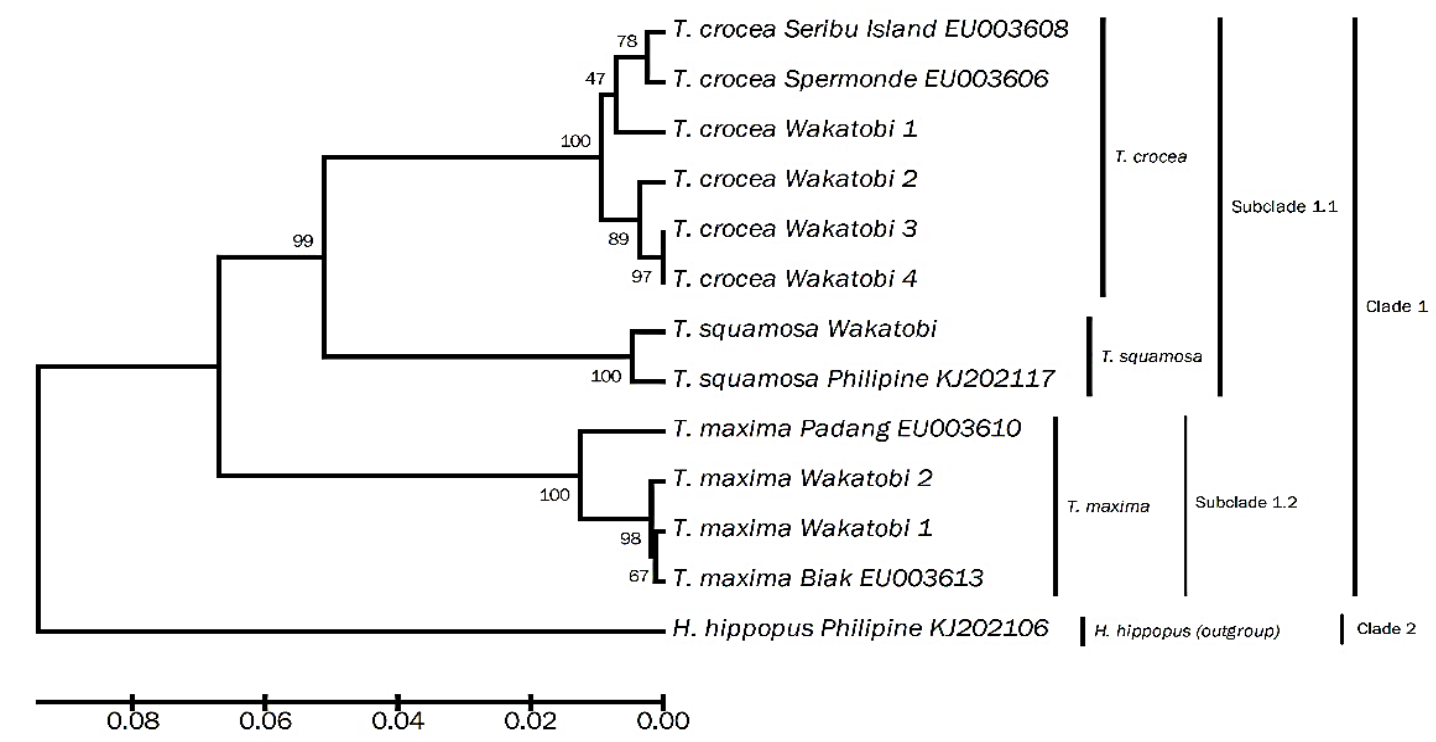

Figure 3. Reconstruction of phylogenetic tree using Neighbour Joining method with p-distance model, 1000 bootstrap replicates.

Table 4. Genetic distance of giant clam COI gene using p-distance model

\begin{tabular}{|c|c|c|c|c|c|c|c|c|c|c|c|c|c|}
\hline & 1 & 2 & 3 & 4 & 5 & 6 & 7 & 8 & 9 & 10 & 11 & 12 & 13 \\
\hline 1 & & & & & & & & & & & & & \\
\hline 2 & 0.019 & & & & & & & & & & & & \\
\hline 3 & 0.021 & 0.007 & & & & & & & & & & & \\
\hline 4 & 0.021 & 0.007 & 0.000 & & & & & & & & & & \\
\hline 5 & 0.012 & 0.016 & 0.019 & 0.019 & & & & & & & & & \\
\hline 6 & 0.016 & 0.016 & 0.019 & 0.019 & 0.005 & & & & & & & & \\
\hline 7 & 0.105 & 0.109 & 0.107 & 0.107 & 0.100 & 0.100 & & & & & & & \\
\hline 8 & 0.100 & 0.105 & 0.102 & 0.102 & 0.095 & 0.095 & 0.009 & & & & & & \\
\hline 9 & 0.133 & 0.137 & 0.130 & 0.130 & 0.133 & 0.133 & 0.142 & 0.142 & & & & & \\
\hline 10 & 0.130 & 0.135 & 0.128 & 0.128 & 0.130 & 0.130 & 0.140 & 0.140 & 0.002 & & & & \\
\hline 11 & 0.128 & 0.133 & 0.130 & 0.130 & 0.128 & 0.128 & 0.137 & 0.137 & 0.023 & 0.026 & & & \\
\hline 12 & 0.135 & 0.140 & 0.133 & 0.133 & 0.135 & 0.135 & 0.144 & 0.144 & 0.002 & 0.005 & 0.026 & & \\
\hline 13 & 0.191 & 0.191 & 0.188 & 0.188 & 0.195 & 0.195 & 0.207 & 0.207 & 0.174 & 0.177 & 0.177 & 0.172 & 0.247 \\
\hline
\end{tabular}

1= T. crocea Wakatobi 1; $2=T$. crocea Wakatobi 2; $3=T$. crocea Wakatobi 3; $4=T$. crocea Wakatobi $4 ; 5=T$. crocea Spermonde EU003606; $6=T$. crocea P. Seribu EU003608; $7=T$. squamosa Wakatobi; $8=T$. squamosa Philipine KJ202117; $9=T$. maxima Wakatobi 1; $10=T$. maxima Wakatobi 2; $11=T$. maxima Padang EU003610; 12 = T. maxima Biak EU003613; $13=H$. hippopus KJ202106 (out group)

Table 5. T. crocea nucleotide polymorphism from Wakatobi, Spermonde and Seribu Island

\begin{tabular}{|c|c|c|c|c|c|c|c|c|c|c|c|c|}
\hline \multirow{2}{*}{ Species } & \multicolumn{12}{|c|}{ Nucleotide position } \\
\hline & 1 & . & . & . & . & 333 & . & . & 360 & . & 366 & . \\
\hline T. crocea Wakatobi 1 & $\mathrm{~T}$ & $\cdot$ & . & . & . & $A$ & . & . & $\mathrm{G}$ & . & $\mathrm{C}$ & . \\
\hline T. crocea Wakatobi 4 & $\mathrm{~T}$ & . & - & . & . & A & . & . & G & . & C & . \\
\hline T. crocea Wakatobi 3 & $\mathrm{~T}$ & ${ }^{\circ}$ & - & . & . & $\mathrm{G}$ & . & . & $A$ & . & $\mathrm{T}$ & . \\
\hline T. crocea Seribu Island GB* & $\mathrm{T}$ & . & . & . & . & $\mathrm{G}$ & . & . & $A$ & . & $\mathrm{T}$ & . \\
\hline
\end{tabular}

*GB = GenBank Data 
Table 6. T. maxima nucleotide polymorphism from Wakatobi, Biak and Padang

\begin{tabular}{lccccccccc}
\hline \multicolumn{1}{c}{ Species } & \multicolumn{10}{c}{ Nucleotide position } \\
\cline { 2 - 20 } & 342 & 171 & 234 & 237 & 243 & 285 & 294 & 312 & 327 \\
\hline T. maxima Wakatobi 1 & $\mathrm{C}$ & $\mathrm{T}$ & $\mathrm{A}$ & $\mathrm{C}$ & $\mathrm{T}$ & $\mathrm{C}$ & $\mathrm{T}$ & $\mathrm{C}$ & $\mathrm{C}$ \\
T. maxima Wakatobi 2 & $\mathrm{C}$ & $\mathrm{T}$ & $\mathrm{A}$ & $\mathrm{C}$ & $\mathrm{T}$ & $\mathrm{C}$ & $\mathrm{T}$ & $\mathrm{C}$ & $\mathrm{C}$ \\
T. maxima Biak GB* & $\mathrm{C}$ & $\mathrm{T}$ & $\mathrm{A}$ & $\mathrm{C}$ & $\mathrm{T}$ & $\mathrm{C}$ & $\mathrm{T}$ & $\mathrm{C}$ & $\mathrm{C}$ \\
T. maxima Padang GB* & $\mathrm{T}$ & $\mathrm{C}$ & $\mathrm{C}$ & $\mathrm{T}$ & $\mathrm{C}$ & $\mathrm{T}$ & $\mathrm{C}$ & $\mathrm{T}$ & $\mathrm{T}$ \\
\hline
\end{tabular}

${ }^{*} \mathrm{~GB}=$ GenBank Data

different genetic structure of bivalve (Donrung et al., 2011), even in a long time would result in different morphological forms (Evans and Hoffman, 2012). T. maxima also formed two groups, which $T$. maxima from Wakatobi clustered with $T$. maxima from Biak and separated with $T$. maxima from Padang. It was also caused by a similarity in the nucleotide composition between $T$. maxima from Wakatobi and T. maxima from Biak (Table 6).

Genetic information could be utilized in giant clams resource management. $T$. crocea Wakatobi varying might be used as a genetic resource. In domestication, it could also be used as a broodstock because it was more genetically varied. Likewise, if we would translocate the clam, we could catch $T$. crocea from Wakatobi as the genetic source. According to Yusron (2005), the strategy to increase the biodiversity of a population and to improve decreasing genetic diversity besides reducing the exploitation rate was introduction of new individuals which had higher genetic diversity into the local population.

\section{Conclusion}

There were 41 specific nucleotide sites that became barcode of each species, $T$. crocea, $T$. squamosa and T. maxima had 2, 15, and 24 sites, respectively. Those facilitated uncovering cryptic species phenomenon in this genus. Nucleotide variation of $T$. crocea from Wakatobi was the highest among $T$. crocea from other locations; therefore, it could be used as a genetic source for translocation and domestication.

\section{Acknowledgment}

The research was funded by Indonesian Government through Directorate General of Higher Education (DIKTI), Ministry of Research, Technology and Higher Education. We would like to deeply thank to the Director and staff of Wakatobi National Park for permission and use facilities during the research. Special thanks also are extended to Muhammad
Lukman, La Ode Orba, Aah, Hendrawan, La Ode Almar, La Engka and Alfian Asmara for their help during sample collection.

\section{References}

Bickford, D., Lohman, D.J. , Sodhi, N.S., Ng, P.K.L., Meier, R., Winkler, K., Ingram. K.K., \& Das, I. 2006. Cryptic species as a window on diversity and conservation. Ecol. and Evol.. 22:148-155.

bin Othman, A.S., Goh, G.H.S. \& Todd, P.A.. 2010. The distribution and status of giant clams (Family Tridacnidae), a short review. Raffles Bull Zool. 58(1):103-111.

Calumpong, H.P. (Ed). 1992. The Giant Clam: An Ocean Culture Manual. ACIAR Monograph. Canberra. 68 pp.

Donrung, P., Tunkijjanukij, S., Jarayabhand, P. \& Poompuang, S. 2011. Spatial genetic structure of the surf clam Paphia undulata in Thailand Waters. Zoological Studies. 50(2): 211-219.

Evans, T.G. \& Hoffman, G.E. 2012. Defining the limits of physiological plasticity: how gene expression can assess and predict the consequences of ocean change. Biological Science. 367: 1733-1745.

Findra, M.N. 2010. Komposisi Jenis, Kelimpahan dan Ukuran Kima di Perairan Pulau Tolandono dan Pulau Sawa, Kawasan Taman Nasional Wakatobi. Skripsi. Universitas Hasanuddin. Makassar. $62 \mathrm{pp}$.

Haerul, A. 2014. Karakterisasi Genetik Karang Genus Favites (Faviidae: Scleractinia) di Perairan Kepulauan Spermonde, Sulawesi Selatan. Tesis. Institut Pertanian Bogor. Bogor. 64 pp.

Hajibabaei, M., Singer, G.A.C., Hebert, P.D.N., \& Hickey, D.A. 2007. DNA barcoding: how it complements taxonomy, molecular 
phylogenetics and population genetics. TRENDS in Genetics. 23(4):167-172. doi: 10.1016/j.tig. 2007.02.001.

Hebert, P.D.N., Cywinska, A., Ball, S.L. \& deWaard, J.R. 2003. Biological identifications through DNA barcodes. Proceedings of the Royal Society of London. Series B: Biological Sciences. 270(1512):313-321.

Hernawan, U.E. 2012. Taxonomy of Indonesian giant clams (Cardiidae, Tridacninae). Biodiversitas. 13(3):118-123.

Knop, D. 1996. Giant Clams: A Comprehensive Guide to the Identification and Care of Tridacnid Clams. Dahne Verlag. Germany. 255 pp.

Lizano, A.M.D. \& M.D. Santos. 2014. Updates on the status of giant clams Tridacna spp. and Hippopus hippopus in the Philippines using mitochondrial C01 and 16S rRNA genes. Philippine Science Letters. 7(1):187-199.

Larrue, S. 2006. Giant clam fishing on the Island of Tubuai, Austral Islands group: between local portrayals, economic necessity and ecological realities. SPC Traditional Marine Resource Management and Knowledge Information Bulletin. (19):3-40.

Lucas, J.S., Ledua, E. \& Braley, R.D. 1990. A new species of giant clam (Tridacnidae) from Fiji and Tonga. Australian Centre for International Agricultural Research. Working Paper. 33:1-8.

Mudjiono. 1988. Catatan beberapa aspek kehidupan kima, Suku Tridacnidae. Oseana. 13(2):37-47.

Nuryanto, A., Duryadi, D., Soedharma, D., \& Blohm, D. 2007. Molecular phylogeny of giant clam based on mitochondrial DNA Cytochrome C Oxidase I Gene. Hayati J. Biosci. 14(4):162166.

Panggabean, L.M.G. 1991. Rahasia kehidupan kima: III. Kelangsungan hidup. Oseana. 16(2):35-45.

Penny, S.S. \& Willan, R.C. 2014. Description of a new species of giant clam (Bivalvia:
Tridacnidae) from Ningaloo Reef, Western Australia. Mollus. Res. 34:201-211.

Ratnasingham, S. \& Hebert, P.D.N. 2013. A DNAbased registry for all animal species: the barcode index number (BIN) system. Plos One. 8(8):e66213.

Richter, C., Roa-Quiaoit, H., Jantzen, C., Al-Zibdah, M. $\&$ Kochzius, M. 2008. Collapse of a new living species of giant clam in the Red Sea. Curr. Biol. 18:1349-1354.

Romimohtarto, K. \&. Juwana, S., 2005. Biologi Laut. Djambatan. Jakarta. 540 pp.

Shau-Hwai A.T. \& Z. Yasin. 2003. Status of giant clams in Malaysia. SPC Trochus Information Bulletin. 10:9-10.

Sirenko, B.I. \& Scarlato, O.A.. 1991. Tridacna rosewateri sp.n. A new species of giant clam from the Indian Ocean. La Conchiglia. 22(261):4-9.

Schneider, J.A. \& O`Foighil, D.1999. Phylogeny of giant clams (cardiidae: tridacninae) based on partial mitochondrial 16S rDNA gene sequences. Mol. Phylog. Evol. 13(1):59-66.

Solihin, D.D. 1994. Peran DNA mitokondria (mtDNA) dalam studi keragaman genetik dan biologi populasi pada hewan. Hayati. 1(1):1-4.

Tamura, K., Peterson, D., Peterson, N., Stecher, G., Nei, M. \& Kumar, S. 2011. MEGA5: Molecular evolutionary genetics analysis using maximum likelihood, evolutionary distance, and maximum parsimony methods. Mol. Biol. Evol. 28(10):2731-2739.

Tisera, W.I., Naguit, M.R.A. Rehatta, B.M. \& Calumpong, H.P. 2012. Ecology and genetic structure of giant clams around Savu Sea, East Nusa Tenggara Province, Indonesia. Asian J. Biodiversity. 10:174-194.

Yusron, E. 2005. Pemanfaatan keragaman genetik dalam pengelolaan sumberdaya hayati laut. Oseana, 30(2):29-34. 